\title{
Superadiabaticity in Jupiter and Giant Planet Interiors
}

\author{
Florian Debras ${ }^{1,2,3}$, Gilles Chabrier ${ }^{2,3}$, and David J. Stevenson ${ }^{4}$ (1) \\ ${ }^{1}$ IRAP, Université de Toulouse, CNRS UMR 5277, UPS, Toulouse, France; florian.debras@irap.omp.eu \\ ${ }^{2}$ Ecole normale supérieure de Lyon, CRAL, UMR CNRS F-5574, 69364 Lyon Cedex 07, France \\ ${ }^{3}$ School of Physics, University of Exeter, Exeter EX4 4QL, UK \\ ${ }^{4}$ Division of Geological and Planetary Sciences, California Institute of Technology, 1200 E California Boulevard, Pasadena, CA 91125, USA \\ Received 2020 October 16; revised 2021 April 22; accepted 2021 May 3; published 2021 May 28
}

\begin{abstract}
Interior models of giant planets traditionally assume that at a given radius (i.e., pressure) the density should be larger than or equal to the one corresponding to a homogeneous, adiabatic stratification throughout the planet (referred to as the "outer adiabat"). The observations of Jupiter's gravity field by Juno combined with the constraints on its atmospheric composition appear to be incompatible with such a profile. In this Letter, we show that the above assumption stems from an incorrect understanding of the Schwarzschild-Ledoux criterion, which is only valid on a local scale. In order to fulfill the buoyancy stability condition, the density gradient with pressure in a nonadiabatic region must indeed rise more steeply than the local adiabatic density gradient. However, the density gradient can be smaller than the one corresponding to the outer adiabat at the same pressure because of the higher temperature in an inhomogeneously stratified medium. Deep enough, the density can therefore be lower than the one corresponding to the outer adiabat. We show that this is permitted only if the slope of the local adiabat becomes shallower than the slope of the outer adiabat at the same pressure, as found in recent Jupiter models due to the increase of both specific entropy and adiabatic index with depth. We examine the dynamical stability of this structure and show that it is stable against nonadiabatic perturbations. The possibility of such an unconventional density profile in Jupiter further complicates our understanding of the internal structure and evolution of (extrasolar) giant planets.
\end{abstract}

Unified Astronomy Thesaurus concepts: Planetary interior (1248); Exoplanet structure (495)

\section{Introduction}

The combination of recent observational and theoretical significant advances, namely, Juno's determinations of Jupiter's high-order gravity moments (Bolton et al. 2017; Iess et al. 2018) and numerical calculations of relevant dense matter equations of state (e.g., Militzer \& Hubbard 2013; Chabrier et al. 2019; Mazevet et al. 2019; Chabrier \& Debras 2021) have drastically impacted our understanding of Jupiter and thus of giant planets in general (Wahl et al. 2017; Debras \& Chabrier 2019).

Recent models with an adiabatic, homogeneous envelope predict a solar or even subsolar heavy element abundance in the atmosphere, a striking likely disagreement with observations. In reality the atmosphere is supersolar, at least based on methane (Wong et al. 2004), and could plausibly be 2 or even 3 times solar if water (the most important heavy element contributor) is also supersolar by a similar amount. If the right equation of state is in use, then it is tempting to attribute the low density in the models to either superadiabaticity combined with a stable compositional gradient or subadiabaticity combined with a unstable compositional gradient. Debras \& Chabrier (2019) proposed the former case of superadiabaticity to reconcile the relatively modest values of the high-order gravitational moments observed by Juno and the high, supersolar heavy element fraction revealed by Galileo. This (counterintuitive) solution, however, raises an important issue. Indeed, according to the Schwarzschild-Ledoux criterion, for a region to be stable against overturning convection, any departure from adiabaticity should lead to a steeper density gradient, thus implying a larger density at depth than the one of the outer adiabat. ${ }^{5}$ Therefore, a structure of Jupiter less dense with depth than the external adiabat needs to be justified on physical grounds, both in terms of stability and of a plausible cooling history.

In this Letter, we derive simple physical arguments to assess the validity of such a density profile. First, we recall in Section 2 the required conditions for a nonadiabatic region to persist in a planet's interior. In Section 3, we show that the the local adiabatic density gradient in the nonadiabatic region must become flatter than the density gradient of the outer adiabat at the same pressure. We show that this condition is fulfilled in recent Jupiter models because of $\mathrm{H}_{2}$ pressure dissociation and atomic $\mathrm{He}$ enrichment at the expense of molecular $\mathrm{H}_{2}$, which both yield a decrease of the number of degrees of freedom and therefore an increase of the adiabatic index. Then, in Section 4 we examine the dynamical stability of such density structures. We briefly examine the type of nonadiabatic evolution for the planet in Section 5. Section 6 is devoted to the conclusion. In spite of their relative simplicity and needing further numerical simulations of much greater complexity to be fully validated, these calculations provide physically sound arguments to justify the possibility of nonadiabatic temperature and density stratifications in Jupiter and giant planet interiors.

\footnotetext{
5 We define the "outer" or "external" isentrope/adiabat as the densitypressure and temperature-pressure profiles Jupiter would have if the specific entropy was constant throughout the planet, at the value inferred from the Galileo and Juno observations, i.e., 1 bar at $166 \mathrm{~K}$ (Wong et al. 2004; Li et al. 2017). We tested that choosing $170 \mathrm{~K}$ or even $175 \mathrm{~K}$ does not change the overall picture (Leconte et al. 2017; Guillot et al. 2020a, 2020b).
} 


\section{Conditions for a Nonadiabatic Region}

For a given composition, associated with a mean molecular weight $\mu$, the thermodynamic properties of the fluid can be calculated from the knowledge of two of the following quantities: specific entropy $S$, temperature $T$, pressure $P$, and mass density $\rho$. Notably, $\rho$ can be calculated from $P$ and $S$ to derive Jupiter models with the concentric MacLaurin spheroid method (Hubbard 2013; Debras \& Chabrier 2018).

Under Jupiter conditions, where motions are small compared to the speed of sound, an adiabat can be approximated as an isentrope $(d Q=0 \leftrightarrow d S=0)$, at least in the absence of irreversible processes such as, e.g., phase separation. In regions where the molecular weight is constant the temperature gradient in the planet is thus equal to the adiabatic temperature gradient (Hubbard 1968; Saumon et al. 1992; Hubbard et al. 2002), defined as

$$
\nabla_{T_{(\mu=c t)}} \equiv\left(\frac{d \ln T}{d \ln P}\right)_{\mu=c t e} \simeq\left(\frac{d \ln T}{d \ln P}\right)_{\mathrm{ad}} \equiv \nabla_{T_{a d}} .
$$

As in convective regions the specific entropy is constant, this is equivalent to say that the density gradient must obey the condition

$$
\nabla_{\rho_{(\mu=c t e)}} \equiv\left(\frac{d \ln \rho}{d \ln P}\right)_{\mu=c t e} \simeq\left(\frac{d \ln \rho}{d \ln P}\right)_{\mathrm{ad}} \equiv \nabla_{\rho_{\mathrm{ad}}}
$$

The need for a lower density than that of an adiabatic profile requires that, somewhere within the planet, $\nabla_{\rho}<\nabla_{\rho_{\mathrm{ad}}}$, which implies regions of compositional gradients. A regime of double-diffusive instability may then develop due to the competition between (fast) thermal diffusivity and (slow) molecular diffusivity. Such regions are stable to overturning convection, thus stable with respect to the Ledoux criterion, but still unstable to small-scale convection, i.e., unstable w.r.t. the Schwarzchild criterion. Two situations can occur depending on the destabilizing or stabilizing nature of the compositional gradient:

1. In the former case, the molecular weight is decreasing with depth in some part of the planet $(d \mu / d r>0$, i.e., $\nabla_{\mu}=d \ln \mu / d \ln P<0$ ) and can lead to a region of fingering convection;

2. In the second, opposite case, the increasing molecular weight with depth $\left(d \mu / d r<0\right.$, i.e., $\left.\nabla_{\mu}>0\right)$ can trigger oscillatory or, more likely, layered convection, possibly under the form of blurred double-diffusive convection, a regime generally identified as "semi-convection" (Mirouh et al. 2012; Moll et al. 2016).

These two situations can be described by the two following respective conditions (e.g., Rosenblum et al. 2011):

$$
\begin{gathered}
\text { fingering convection: } 1<R_{\rho}<\frac{1}{\tau}, \\
\text { semi-convection: } \quad 1<R_{\rho}^{-1}<\frac{\operatorname{Pr}+1}{\operatorname{Pr}+\tau},
\end{gathered}
$$

where $\operatorname{Pr}=\nu / \kappa_{T}$ and $\tau=\kappa_{\mu} / \kappa_{T}$ denote respectively the Prandtl and inverse Lewis numbers, with $\nu$ the molecular viscosity, $\kappa_{\mu}$ and $\kappa_{T}$ the molecular and thermal diffusivities,

$$
\begin{gathered}
R_{\rho}=\frac{\alpha_{T}}{\alpha_{\mu}} \frac{\nabla_{T}-\nabla_{T_{a d}}}{\nabla_{\mu}} \text { is the density contrast parameter, and } \\
\alpha_{\mu}=\left(\frac{\partial \ln \rho}{\partial \ln \mu}\right)_{T, P}=-\left(\frac{\partial \ln \rho}{\partial \ln P}\right)_{T, \mu}\left(\frac{\partial \ln P}{\partial \ln \mu}\right)_{T, \rho}, \\
\alpha_{T}=-\left(\frac{\partial \ln \rho}{\partial \ln T}\right)_{P, \mu}=\left(\frac{\partial \ln \rho}{\partial \ln P}\right)_{T, \mu}\left(\frac{\partial \ln P}{\partial \ln T}\right)_{\rho, \mu}
\end{gathered}
$$

only depend on the equation of state (EOS). For any realistic EOS in Jupiter, $\alpha_{T}>0$ and $\alpha_{\mu}>0$.

These conditions can be rewritten in terms of density, instead of temperature. For a general $\operatorname{EOS} P(\rho, T, \mu)$, it is easy to show that these conditions become

fingering convection:

$$
\nabla_{\rho_{\mathrm{ad}}}<\nabla_{\rho}<\nabla_{\rho_{\mathrm{ad}}}+\left(\frac{1}{\tau}-1\right) \frac{\chi_{\mu}}{\chi_{\rho}} \nabla_{\mu},
$$

semi-convection:

$$
\nabla_{\rho_{\mathrm{ad}}}<\nabla_{\rho}<\nabla_{\rho_{\mathrm{ad}}}-\left(\frac{1-\tau}{1+P r}\right) \frac{\chi_{\mu}}{\chi_{\rho}} \nabla_{\mu}
$$

where

$$
\begin{aligned}
& \chi_{\mu}=\left(\frac{\partial \ln P}{\partial \ln \mu}\right)_{T, \rho}, \\
& \chi_{\rho}=\left(\frac{\partial \ln P}{\partial \ln \rho}\right)_{\mu, T} .
\end{aligned}
$$

Since under the conditions of interest $\tau \approx 10^{-2} \ll 1, \operatorname{Pr} \approx$ $10^{-2}-10^{-1}$ (see, e.g., Chabrier \& Baraffe 2007), $\chi_{\mu}<0$, $\chi_{\rho}>0$, and $\nabla_{\mu}<0$ and $>0$, respectively, in the fingering and semi-convection case, these inequalities can be fulfilled.

The left-hand side of the two inequalities expresses the same property: in a system stable to overturning convection, the density of a parcel of fluid raised adiabatically to a lower pressure must be larger than that of its new surroundings. The right-hand side conditions correspond to the stratification limits below which the regions are stable w.r.t the Ledoux criterion but unstable to small-scale double-diffusive instability.

A region of fingering convection in the outermost layers, i.e., $\nabla_{\mu}<0$, may occur either because of (i) accretion of hot entropy gas during the runaway gas accretion phase, hampering or even completely inhibiting convection in the outermost part of the planet (Berardo et al. 2017; Berardo \& Cumming 2017), and/or (ii) the coaccretion of rock and gas or accretion of planetesimals over some fraction of the planet's surface during the planet's history (e.g., Iaroslavitz \& Podolak 2007; Mordasini et al. 2017).

In this Letter, we only consider a region of increasing molecular weight with depth $\left(d \mu / d r<0\right.$, i.e., $\left.\nabla_{\mu}>0\right)$ as there is a need for such a region in Jupiter's interior. Indeed, Galileo's observations of helium atmospheric abundance (von Zahn et al. 1998) reveal only $90 \%$ of the protosolar value inferred from solar models (e.g., Anders \& Grevesse 1989), whereas the global helium content of Jupiter is presumably protosolar. This increase of helium mass fraction with depth yields an increase of molecular weight. Such a gradient could be due either to $\mathrm{H} / \mathrm{He}$ immiscibility, leading to helium sedimentation (Stevenson \& Salpeter 1977; Stevenson 1979; Fortney \& Hubbard 2003), or to a region of layered or blurred double-diffusive convection, possibly triggered by 


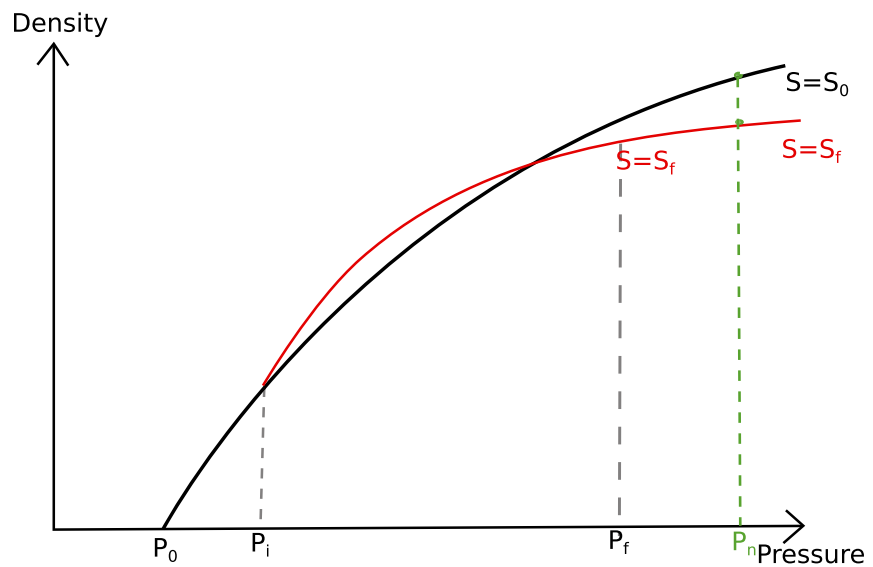

Figure 1. Cartoon of the superadiabatic profile compared with an adiabatic one. The overdense region compared to the adiabat is exaggerated for illustration purposes.

immiscibility (see the discussions in Stevenson \& Salpeter 1977; Debras \& Chabrier 2019). It is worth noting that semiconvection occurs more easily in regions where $\left|\chi_{\mu} / \chi_{\rho}\right|>1$ (see Equation (8)), hence deeper than $1 \mathrm{GPa}(=10 \mathrm{kbar}$; Debras \& Chabrier (2019)).

At first sight, the conditions (7) and (8) seem to contradict the recent models of Debras \& Chabrier (2019) since the first obvious conclusion is that the density gradient must be steeper than the adiabatic density gradient. The correct analysis, however, is more subtle. In a convective homogeneous medium, the adiabatic profile can be considered as a global profile and the specific entropy is constant throughout the entire region. In a medium prone to composition change, however, $\nabla_{\rho_{\text {ad }}}$ is only defined on a local scale, as the specific entropy changes with depth. Therefore, the density gradient can be always steeper than the local adiabatic density gradient, defined by the thermodynamical properties of the medium at a given pressure, although flatter than the density gradient the outer adiabat would have at the same pressure. This issue is explored in detail in the following sections.

In all cases, this implies an intermediate inhomogeneous region departing from an adiabatic profile, bracketed by two adiabatic ones in Jupiter's gas-rich envelope. According to the analysis of Debras \& Chabrier (2019) using state-of-the art H/He EOS (Chabrier et al. 2019; Chabrier \& Debras 2021), sufficiently deep in this region the density should be lower than the density of the external isentrope at the same pressure.

\section{Thermodynamic Considerations}

Figure 1 displays a $\rho(P)$ profile where we suppose the existence of a superadiabatic region between pressures $P_{i}$ and $P_{f}$, associated with a decrease in density at depth (red line), compared with the outer adiabatic profile (black line). The outer and inner specific entropies are $S=S_{0}$ at $P_{i}$ and $S=S_{f}$ at $P_{f}$, respectively.

The nonadiabatic region can be characterized by two physical processes: a diffusive region or a semi-convective region. In both cases, the left-hand side of Equation (8) must be verified: the density gradient must be steeper than the density gradient of the local adiabat. However, Figure 1 shows that we want the density gradient to be shallower than the one of the

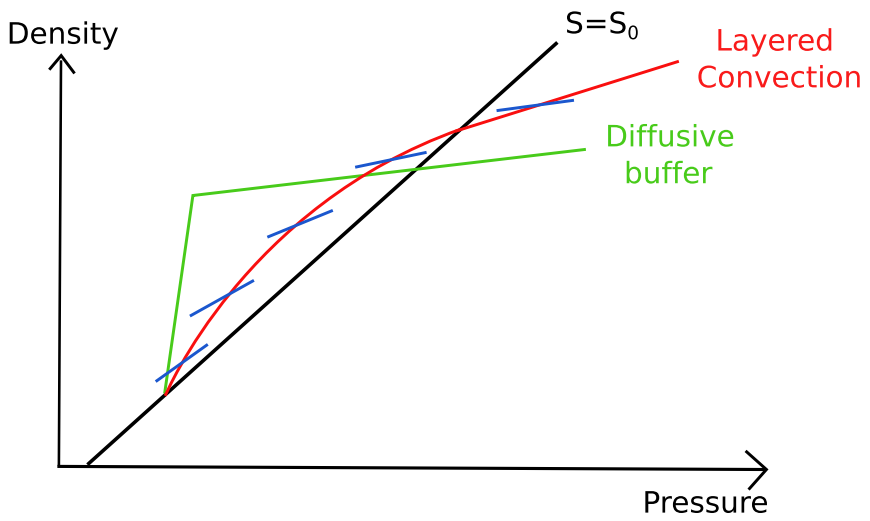

Figure 2. Cartoon of $P-\rho$ profiles for isentropic profile (black), semiconvection region (red), and diffusive buffer (green). The blue lines are the local isentropes in the semi-convective profile. Comparing black with red or green lines, the diffusive or layered convective profiles exhibit initially a steeper density gradient than the isentropic profile, but become eventually less dense at depth because of the decreasing steepness of the density gradient.

outer adiabat. Therefore, the nonadiabatic region must verify

$$
\left.\frac{d \rho}{d P}\right|_{S}<\frac{d \rho}{d P}<\left.\frac{d \rho}{d P}\right|_{S_{0}},
$$

where $d \rho /\left.d P\right|_{S}$ is the adiabatic density gradient at pressure $P$ in the region, $d \rho / d P$ is the actual density gradient, and $d \rho /\left.d P\right|_{S_{0}}$ is the density gradient of the outer adiabat at the same pressure $P$.

It is easily shown that for an adiabatic reversible transformation $(d Q=T d S=0)$, the density gradient must verify

$$
\left.\frac{d \rho}{d P}\right|_{S}=\gamma^{-1}\left(\frac{\partial \rho}{\partial P}\right)_{T}
$$

where $\gamma=C_{P} / C_{V}$ is the usual adiabatic index, i.e., ratio of the specific heats at constant pressure and volume, respectively. For an ideal gas, this relation yields the Laplace law. For a general EOS, $\gamma$ depends on the thermodynamic quantities. As the temperature is a function of pressure and entropy in our models, Equation (10) then implies a condition on the local adiabatic density gradient $d \rho /\left.d P\right|_{S}$, namely,

$$
\gamma^{-1}(P, S)\left(\frac{\partial \rho}{\partial P}\right)_{T}(P, S)<\gamma^{-1}\left(P, S_{0}\right)\left(\frac{\partial \rho}{\partial P}\right)_{T_{0}}\left(P, S_{0}\right)
$$

If condition (12) is satisfied in the nonadiabatic regions of Jupiter, the density gradient can then become flatter than the one of the outer adiabat. Eventually, the density of the nonadiabatic structure can thus become lower than the density of the outer adiabat at the same pressure. Note that, because of the superadiabiticity in the semi-convection zone, each layer lies on a warmer isentrope than the outer one, $T(P, S)>T_{0}(P$, $\left.S_{0}\right)$. This contributes to decreasing $(\partial \rho / \partial P)_{T}$.

Such a profile is illustrated in Figure 2 for two cases: a single, small diffusive buffer or an extended layered convection region. At the bottom of the diffusive buffer, the adiabatic gradient is much flatter than the gradient of the outer adiabat because of the sharp increase in temperature. The semiconvective region, on the other hand, is characterized by a slow flattening of the local adiabatic gradients with depth. In both 

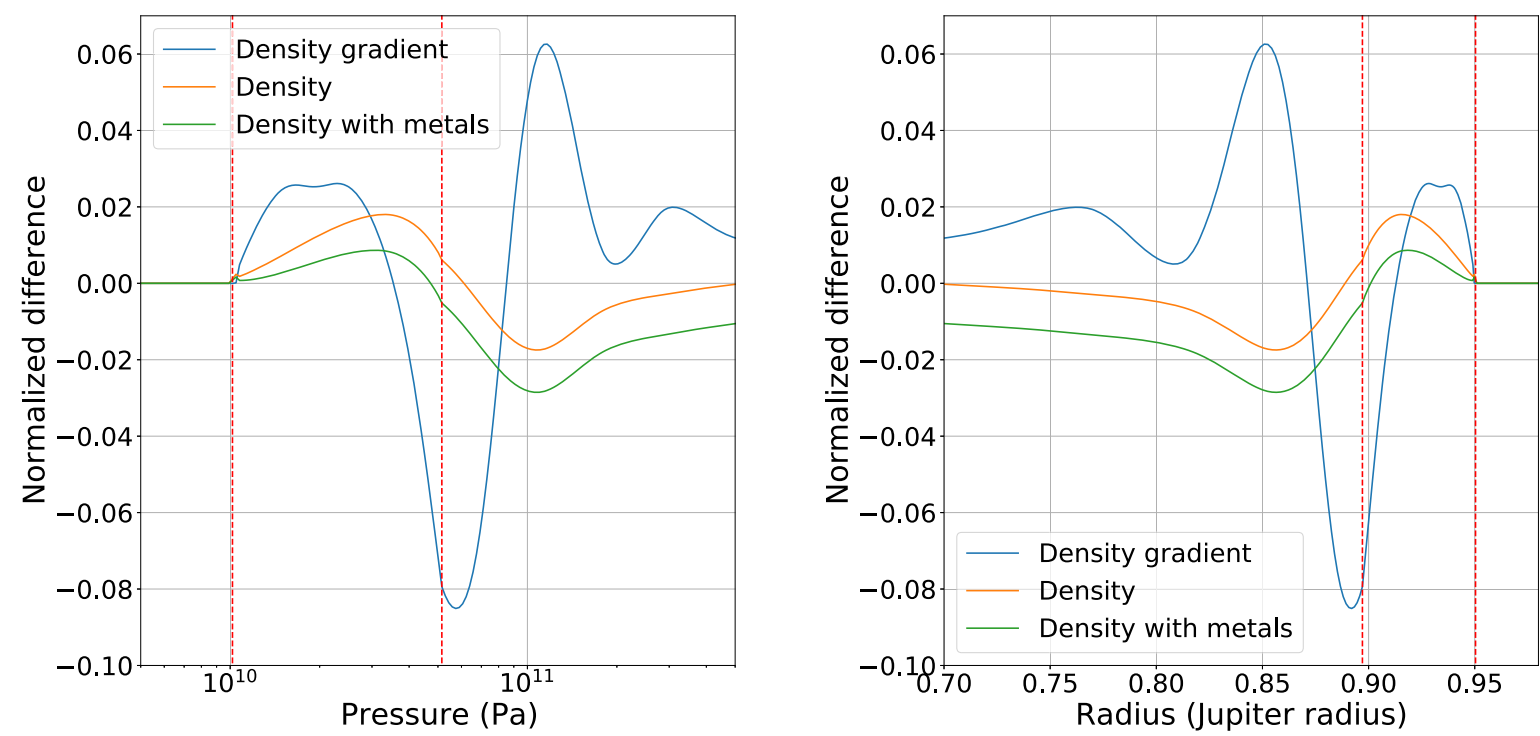

Figure 3. Blue curves: difference between the local adiabatic density gradient from a model of Debras \& Chabrier (2019) and the density gradient of the outer adiabat at the same pressure (left) or radius (right), divided by the density gradient of the outer adiabat. Orange curves: difference between density of the model and the density of the outer adiabat, divided by the density of the outer adiabat. Green curves: same as orange curve, but including the decrease of metal content with depth as proposed in Debras \& Chabrier (2019). Red vertical dashed lines: limits of the semi-convective zone, separating the two convective envelopes. Note: $1 \mathrm{Mbar}=10^{11} \mathrm{~Pa}$.

cases, the density eventually becomes lower than the density of the outer adiabat at depth. ${ }^{6}$

In Figure 3, we show the difference between the local adiabatic density gradient and the density gradient of the outer adiabat in a typical model of Debras \& Chabrier (2019), as well as the difference between the true density and the one of the outer adiabat, as a function of pressure and radius, respectively. We see that there is a direct correlation between the two curves: when Equation (12) is verified (blue curve negative), the density decreases compared to the density of the outer adiabat (orange/green curve decreasing) and, inversely, confirming the validity of the model. The fact that Equation (12) is fullfilled in the semi-convection region of Jupiter $(P \simeq 10-100 \mathrm{GPa})$ stems from several factors. First, as mentioned above, the superadiabaticity decreases the local density gradient, $(\partial \rho / \partial P)_{T}$. Second, the adiabatic index $\gamma$ increases with depth because of (i) $\mathrm{H}_{2}$ pressure dissociation and (ii) atomic $\mathrm{He}$ enrichment at the expense of molecular $\mathrm{H}_{2}$, which both yield a decrease of the number of degrees of freedom.

We see in Figure 3 that the density is larger than the density of the outer adiabat between 0.1 and $0.6 \mathrm{Mbar}$, i.e., about $4000 \mathrm{~km}$, which encompasses the whole semi-convective region. This result will be used in the next section.

\section{Conditions for Dynamical Stability}

\subsection{Buoyancy Arguments}

In this section, we examine the stability of the pressuredensity profiles mentioned in the previous section, which have been shown to be statically stable, against dynamical perturbations. We first examine the condition of buoyancy stability.

Let us assume that a parcel of fluid from the adiabatic envelope above the semi-convective region can move downward

\footnotetext{
6 Strictly speaking, the pressure at given radius will be slightly different between the fully adiabatic and superadiabatic profiles. However, the difference in the two $P(r)$ profiles, which corresponds to the change of gravity between the two models, is very slow to develop and fairly negligible (see Figure 9 of Debras \& Chabrier 2019).
}

adiabatically over great distances. As examined in Section 2, static stability implies that the parcel must first travel throughout regions that exhibit steeper density gradients than the adiabatic density gradient of the parcel along its path. A simple buoyancy argument gives the deceleration rate of the particle due to the locally, overdense surrounding medium:

$$
\frac{d v}{d t} \sim\left(\frac{\rho_{\mathrm{ad}}-\rho}{\rho_{\mathrm{ad}}}\right) g
$$

where $v$ is the parcel velocity, $g$ the gravitational acceleration, $\rho_{\text {ad }}$ the density of the parcel displaced adiabatically, and $\rho$ the density of the medium. For simplification, let us assume that $\left(\rho_{\mathrm{ad}}-\rho\right) / \rho_{\mathrm{ad}}$ is constant with depth, and $d v / d t \approx v / t_{\mathrm{dec}}$, with $t_{\mathrm{dec}}$ a characteristic deceleration timescale for the parcel. The condition for the particle to travel through a distance $l$ is roughly

$$
\frac{l}{v} \sim t_{\mathrm{dec}} \Rightarrow \quad l \sim \frac{v^{2} \rho_{\mathrm{ad}}}{\left(\rho-\rho_{\mathrm{ad}}\right) g} .
$$

From the mixing length theory (Hansen \& Kawaler 1994; Kippenhahn \& Weigert 1990), one gets for the convective velocity in the $0.1-2$ Mbar region $v \sim 0.1 \mathrm{~m} \mathrm{~s}^{-1}$, and $g \sim$ $10 \mathrm{~m} \mathrm{~s}^{-2}$ for Jupiter, while the typical departure from an adiabatic profile over a few $\mathrm{km} \mathrm{s}^{-1}$ in the models of Debras \& Chabrier (2019) is

$$
\frac{\Delta \rho}{\rho_{\mathrm{ad}}}=\left|\frac{\rho_{\mathrm{ad}}-\rho}{\rho_{\mathrm{ad}}}\right|_{50 \mathrm{~km}} \sim 10^{-4} .
$$

An order of magnitude estimate for the distance $l$ is then

$$
l \sim 10 \mathrm{~m}\left(\frac{v}{0.1 \mathrm{~m} \mathrm{~s}^{-1}}\right)^{2}\left(\frac{\Delta \rho / \rho_{\mathrm{ad}}}{10^{-4}}\right)^{-1}\left(\frac{g}{10 \mathrm{~m} \mathrm{~s}^{-2}}\right)^{-1} .
$$

Hence, for the nonadiabatic region to be dynamically stable, any parcel sinking adiabatically must have a lower density than the local medium over a few tens of meters. The density must 
thus increase more steeply with pressure than the outer adiabatic profile only over $\sim 100 \mathrm{~m}$ or so to prevent the destabilization of the medium.

Examining the onset of layered convection in Jupiter, Chabrier \& Baraffe (2007) and Leconte \& Chabrier (2012) analytically estimated that the typical size of the convective layers in the semi-convection region is $\sim 100 \mathrm{~m}$. Therefore, for the abovementioned typical relative increase in density, of the order of $10^{-4}$, one single layer with a density gradient steeper than the one of the outer adiabat is sufficient for the buoyancy restoring force to stabilize the medium. This is of course a very crude estimate, but it shows that the medium is extremely stable to adiabatic perturbations.

As mentioned in Section 3 and shown in Figure 3, typical profiles in Debras \& Chabrier (2019) exhibit a higher density than the outer adiabat over the whole semi-convective zone, which is $3000 \mathrm{~km}$ large. This encompasses about 30,000 convective layers, which largely fulfill the aforementioned buoyancy stability condition. Two conclusions can then be drawn for the regions of layered convection with a density gradient shallower than the one of the external adiabat at the same pressure:

1. The first layers at the top of the semi-convection zone, where the molecular weight gradient starts to develop, must be denser than the outer adiabat at the same pressure. As shown above, one single convective layer fulfilling this condition is sufficient to stabilize the medium against buoyancy instability.

2 . For the typical departure from the adiabatic density profile found in the models (Equation (15)), a parcel of fluid brought downward adiabatically from some level to higher pressure levels does not become unstable unless its velocity is 2-3 orders of magnitude larger than the convective velocity, because of the buoyancy restoring force.

\subsection{Wave Instability}

Let us now consider an adiabatic, horizontal, divergence-free perturbation of given wavelength $\lambda$ and vertical amplitude $h$. We denote $H$ as the typical height over which the medium becomes less dense than the outer adiabat. The discussion of Section 4.1 shows that $H \gtrsim 100 \mathrm{~m}$ at least, and the models of Debras \& Chabrier (2019) allow for $H \sim 3000 \mathrm{~km}$.

If the initial amplitude of the wave $h \ll H$, the wave satisfies the Schwarzschild criterion at every point: it will be damped out on a buoyant timescale. On the other hand, if $h \gtrsim H$, the antinodes of the wave are buoyantly unstable and the wave may become globally unstable. By analogy with Rayleigh-Taylor instability, rotation and magnetic field might prevent instability to occur. However, using Chandrasekhar (1961), it can be easily verified that under Jupiter's conditions, namely, angular velocity $\Omega \sim 10^{-4} \mathrm{~s}^{-1}$ and magnetic field amplitude of the order of a few tens of Gauss, $B=\mu H \approx 30 \mathrm{G}$ at $r=0.85 R_{\mathrm{J}}$ (Connerney et al. 2018), i.e., at the location of the semiconvective zone (Debras \& Chabrier 2019), rotation and magnetic tension can only stabilize short wavelength $(\lambda \lesssim 100$ $\mathrm{km})$ perturbation waves. The real situation, however, is more complex than described above for three reasons:

(i) The growth timescale of the instability depends in reality on the integral of the density difference with pressure, $\int\left(\rho-\rho_{\mathrm{ad}}\right) v_{z} d P$, where $\rho_{\mathrm{ad}}$ is the density of the outer adiabat and $v_{z}$ denotes the vertical component of the perturbation velocity (Equation (X 44) of Chandrasekhar 1961). As the path along the integral will alternate underdense and overdense regions compared with the outer adiabat, respectively, destabilizing/ stabilizing the medium, it is not clear what the final result will be. At the very least, the growth timescale will be increased compared to a simple Rayleigh-Taylor estimation for two fluids of constant density.

(ii) The wave must remain adiabatic over the entire superadiabatic region to have a chance to destabilize it. This corresponds to $\sim 3000 \mathrm{~km}$ in the models of Debras \& Chabrier (2019). The perturbation must remain adiabatic over this scale since instability can occur only if the growth timescale is shorter than heat transfer timescales. Whereas atomic thermal diffusivity is too small to yield strong departure from adiabaticity $\left(\kappa_{T} \approx 10^{-1} \mathrm{~cm}^{2} \mathrm{~s}^{-1}\right.$ for metallic hydrogen; Stevenson 1977), this is less clear in the case of semiconvection, with significantly enhanced diffusivity and the formation of strong localized updrafts and downdrafts (Rosenblum et al. 2011). This is even more true in the case of a phase separation with a release of latent heat, i.e., $\Delta S \neq 0$.

(iii) An obvious question is what physical mechanism can excite such long wavelength perturbations and what is the typical timescale to excite them? If the perturbation is triggered by convection, the most likely hypothesis, the size of the overshoot plumes at the interface of the double-diffusive region must be comparable to a significant fraction of the size of this latter, i.e., $\sim 3000 \mathrm{~km}$. Assuming rough equipartition between the wave energy and the excitation mechanism (at least in the linear phase), this will roughly correspond to the wave amplitude, which by itself challenges the existence of such "perturbations." Given the typical convective velocity $v \simeq$ $0.1 \mathrm{~m} \mathrm{~s}^{-1}$, it will take about 300 days to generate such a wave. As examined in Section 4.1, damping due to buoyancy occurs on a much shorter timescale. The problem can be rephrased the other way around: there is no obvious physical mechanism susceptible to excite from linear perturbations long wavelength adiabatic perturbations with an initial amplitude of thousands of kilometers at the interface between the external envelope and the inhomogeneous zone in Jupiter's structure models proposed by Debras \& Chabrier (2019).

\section{Evolution}

It is well known that an ongoing adiabatic contraction of Jupiter since its formation yields an age in good agreement with the age of the solar system, as shown originally by Hubbard (1977). Carrying out cooling calculations in the case of a nonadiabatic internal structure cannot be done analytically and requires numerical calculations. Although several attempts have been made to explore this issue, none of them has properly included all the various processes potentially responsible for such a departure from adiabaticity, namely, (i) phase separation, (ii) double-diffusive convection, and (iii) core erosion since recent Jupiter models strongly suggest a diluted core for Jupiter (Wahl et al. 2017; Debras \& Chabrier 2019). Given the complexity of these processes, providing a robust complete cooling history of Jupiter appears to be rather elusive for now, in spite of some claims in the literature. Indeed, besides the uncertainty in existing $\mathrm{H} / \mathrm{He}$ phase diagram calculations, by itself a major source of uncertainty, there is a trade off between these various mechanisms, which can either increase or decrease the cooling rate of the planet, depending whether, and when, they lead to a production or a loss of energy. A typical example, for instance, are the calculations of Leconte \& Chabrier (2013), which show that the correct age 


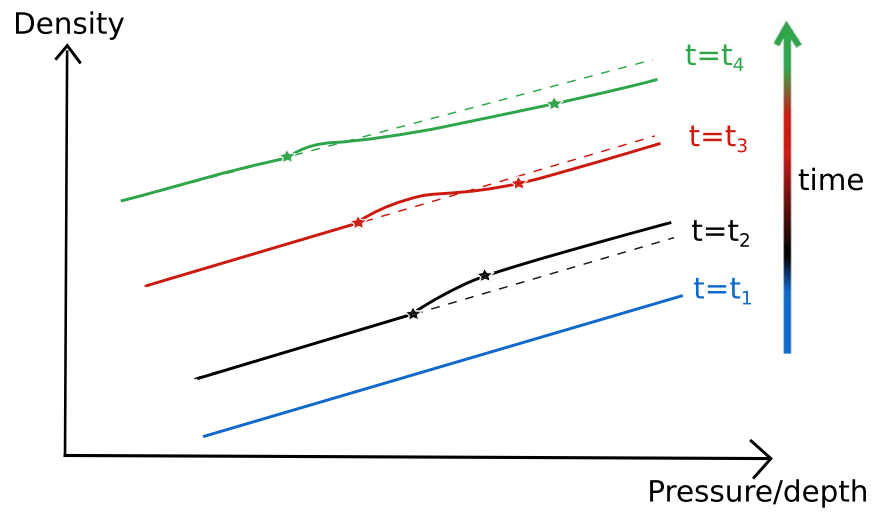

Figure 4. Possible evolutionary path for Jupiter, as outlined in Section 5. Straight dashed lines correspond to fully isentropic profiles; curved solid lines illustrate the nonadiabatic pressure-density structure. The stars bracket the nonadiabatic zone in the Mbar region. At $t=t_{1}$ (blue), Jupiter is fully isentropic; dashed and plain lines are identical. At $t=t_{2}$ (black), the planet has cooled down, immiscibility of hydrogen and helium or semi-convection has begun, and Rayleigh-Taylor instability requires that the inner density profile must be denser than the adiabatic one. At $t=t_{3}$ (red), the nonadiabatic region has expanded, and the superadiabatic temperature profile has steepened, leading to a lower density at higher depth than the external isentrope. At $t=t_{4}$ (green), the overdense region at the top of the semi-convection region has shrunk and almost the whole internal envelope exhibits a density smaller than the outer adiabat would have. The dilution of the core, however, might lead to a higher density than the adiabatic planet in the innermost part of the planet (see Debras \& Chabrier 2019). At this stage, the luminosities of the adiabatic and nonadiabatic planets are comparable.

and luminosity of Saturn can be obtained in the case of layered convection. The reason is that a nonadiabatic region decreases the output heat flux between the interior and the photosphere compared with an entirely convective planet, yielding a decrease of the luminosity. As the energy transport is less efficient, however, a nonadiabatic planet eventually becomes more luminous than an adiabatic one, as it cools down over longer timescales. For Saturn, in the case of the calculations of Leconte \& Chabrier (2013), the crossover happens after a few hundred millions of years (their Figure 2). Layered convection or immiscibility happening about a few hundred millions years ago in Jupiter's interior, leading to a nonisentropic internal region, could thus very well be consistent with the planet's current luminosity. Not to mention, again, the impact of core erosion on the energy balance (Stevenson 1985; Guillot et al. 2004).

A plausible, qualitative evolutionary path for the planet, consistent with both its present luminosity and stability conditions, could thus be as follows, as shown schematically in Figure 4:

1. Formation of Jupiter as a compact core and a convective envelope. Whether the original envelope is well homogenized or not is not really consequential, as inhomogeneous regions could occur during the formation process or later on during the evolution (see Section 2).

2. After a certain time, the temperature in the Mbar region is sufficiently low for helium immiscibility to occur, possibly with other elements as well. Helium droplets begin to drown, depleting the outer envelope in helium and possibly enriching it modestly in heavy elements (Stevenson \& Salpeter 1977; Debras \& Chabrier 2019). As examined in Section 3, initially, the inner density profile must be denser than the adiabatic profile to ensure stability. The demixing process very likely triggers a double-diffusive instability, either as a steady state or constantly dynamically generated by gravity waves at the interface with the convective envelope. Double-diffusive convection, however, can very well occur before (i.e., in the absence of) $\mathrm{H} / \mathrm{He}$ demixion, notably if external impacts hampered the convective efficiency at some epoch during Jupiter's lifetime. Once an external nonadiabatically stratified region has developed, splitting the outer envelope into two separated convective zones, accretion of small solid bodies by Jupiter during its subsequent evolution could lead to the observed supersolar abundance of heavy elements, whatever the initial impact of such a semi-convective zone upon the heavy elements. Accreting between 0.3 and 1.5 Earth mass of heavy elements, depending on various assumptions, over Jupiter's cooling history would fullfill this constraint, which seems to be a plausible hypothesis (see also Podolak et al. 2020).

3. Either process yields the presence of an extended inhomogeneous (nonadiabatic) region around the Mbar level or so. The outer envelope is depleted in helium and enriched in heavy elements, and cools efficiently. In contrast, the inner envelope, slightly enriched in helium, cannot efficiently evacuate its heat content and thus develops a superadiabatic temperature profile, as discussed in Section 4. The luminosity of Jupiter is then lower than for a purely adiabatic contraction. As examined in this Letter, the density in the superadiabatic region can be smaller than the density the outer adiabatic profile would have at the same radius, a condition that seems to be required to fullfill the gravity field constraints (Debras \& Chabrier 2019).

4. At later stages, the outer envelope has cooled down further and then the heat flux of the inner envelope is released more efficiently: the planet then cools more quickly than a fully adiabatic planet (see Figure 2 of Leconte \& Chabrier 2013). Somehow adding up the work required to dilute the core at the expense of convection, the nonadiabatic planet can eventually have a luminosity today consistent with Jupiter's present luminosity.

\section{Conclusion}

In this work, we have explored the consequences of the onset of nonadiabatic temperature and density stratifications in Jupiter and, more generally, in gaseous giant planet interiors. We have explored the possibility of decreasing the steepness of the density gradient with pressure compared with the one of the adiabat of uniform composition. A nonadiabatic region is necessarily characterized by a change in the mean molecular weight $\mu$ with depth, and we have focused on the case of semiconvection, where $\mu$ increases with depth.

On a local scale, we have shown that the density gradient must always be steeper than the local adiabatic density gradient. However, if the slope of the adiabatic density gradient decreases with depth in the nonadiabatic region, deep in the planet this gradient can become flatter than the one the outer adiabat would have at the same pressure. The density at depth can then very well be lower than the density of the outer adiabat at the same pressure. Such a structure requires a decrease of $\gamma^{-1}(\partial \rho / \partial P)_{T}$ compared with the outer adiabat (see Equation (12)), a condition fulfilled in typical models of 
Debras \& Chabrier (2019). This decrease arises essentially from the superadiabatic temperature stratification and, potentially, from hydrogen pressure dissociation and/or hydrogenhelium phase separation.

We have shown that the medium is statically stable, and dynamically stable against nonadiabatic or low-amplitude adiabatic perturbations. Only adiabatic perturbations of large initial amplitude $(\gtrsim 1000 \mathrm{~km})$ could destabilize the medium, but we did not find a plausible origin and a physical justification for the existence of such perturbations.

Although hydrostatic calculations do not enable us to explore in detail such dynamical considerations, the present analysis provides constraints at the kilometer scale on Jupiter's inner structure.

Finally, we have shown that the planet evolution does not provide strong enough constraints on the inner structure profile. Indeed, given the complexity of the various possible physical processes in the interior of gaseous planets (helium rain, semiconvection, core dilution, etc.), and our ignorance of their proper description, reliable evolutionary calculations for the planet remain out of reach for now.

All in all, this Letter demonstrates the possibility of stable superadiabatic regions in Jupiter and giant planets, where the density is smaller than the density the outer adiabat would have at the same radius. Although numerous questions remain open to fully validate these models, high-quality observations coupled with state-of-the-art equations of state for dense matter have drastically changed our understanding of giant planet structure and evolution, with major consequences for extrasolar planets in general. Notably, the present calculations confirm that:

1. There is no correlation between the observed external abundance of heavy elements and the planet's bulk composition as convection can be hampered early in the planet's history;

2. The cooling of gaseous planets can be much more complex than the standard paradigm of homogeneous, adiabatic contraction.

The authors are thankful to Christoph Mordasini, Jérémy Leconte, and Etienne Jaupart for helpful conversations. We are also grateful to the referee whose detailed report greatly helped us in improving the manuscript. This work was supported by the Programme National de Planetologie (PNP) of CNRSINSU co-funded by CNES. F.D. thanks the European Research Council (ERC) for funding under the H2020 research \& innovation program (grant agreement \#740651 NewWorlds).

\section{ORCID iDs}

David J. Stevenson @ \ttps://orcid.org/0000-0001-9432-7159

\section{References}

Anders, E., \& Grevesse, N. 1989, GeCoA, 53, 197

Berardo, D., \& Cumming, A. 2017, ApJL, 846, L17

Berardo, D., Cumming, A., \& Marleau, G.-D. 2017, ApJ, 834, 149

Bolton, S. J., Adriani, A., Adumitroaie, V., et al. 2017, Sci, 356, 821

Chabrier, G., \& Baraffe, I. 2007, ApJL, 661, L81

Chabrier, G., \& Debras, F. 2021, ApJ, in press

Chabrier, G., Mazevet, S., \& Soubiran, F. 2019, ApJ, 872, 51

Chandrasekhar, S. 1961, Hydrodynamic and Hydromagnetic Stability (Oxford: Clarendon Press)

Connerney, J. E. P., Kotsiaros, S., Oliversen, R. J., et al. 2018, GeoRL, 45,2590

Debras, F., \& Chabrier, G. 2018, A\&A, 609, A97

Debras, F., \& Chabrier, G. 2019, ApJ, 872, 100

Fortney, J. J., \& Hubbard, W. B. 2003, Icar, 164, 228

Guillot, T., Li, C., Bolton, S. J., et al. 2020a, JGRE, 125, e06404

Guillot, T., Stevenson, D. J., Atreya, S. K., Bolton, S. J., \& Becker, H. N. 2020b, JGRE, 125, e06403

Guillot, T., Stevenson, D. J., Hubbard, W. B., \& Saumon, D. 2004, in The Interior of Jupiter, ed. F. Bagenal, T. E. Dowling, \& W. B. McKinnon (Oxford: Oxford Univ. Press), 35

Hansen, C. J., \& Kawaler, S. D. 1994, Stellar Interiors. Physical Principles, Structure, and Evolution, Vol. 84 (New York: Springer)

Hubbard, W. B. 1968, ApJ, 152, 745

Hubbard, W. B. 1977, Icar, 30, 305

Hubbard, W. B. 2013, ApJ, 768, 43

Hubbard, W. B., Burrows, A., \& Lunine, J. I. 2002, ARA\&A, 40, 103

Iaroslavitz, E., \& Podolak, M. 2007, Icar, 187, 600

Iess, L., Folkner, W. M., Durante, D., et al. 2018, Natur, 555, 220

Kippenhahn, R., \& Weigert, A. 1990, Stellar Structure and Evolution, Vol. 192 (New York: Springer)

Leconte, J., \& Chabrier, G. 2012, A\&A, 540, A20

Leconte, J., \& Chabrier, G. 2013, NatGe, 6, 347

Leconte, J., Selsis, F., Hersant, F., \& Guillot, T. 2017, A\&A, 598, A98

Li, C., Ingersoll, A., Janssen, M., et al. 2017, GeoRL, 44, 5317

Mazevet, S., Licari, A., Chabrier, G., \& Potekhin, A. Y. 2019, A\&A, 621, A128

Militzer, B., \& Hubbard, W. B. 2013, ApJ, 774, 148

Mirouh, G. M., Garaud, P., Stellmach, S., Traxler, A. L., \& Wood, T. S. 2012, ApJ, 750, 61

Moll, R., Garaud, P., \& Stellmach, S. 2016, ApJ, 823, 33

Mordasini, C., Marleau, G.-D., \& Mollière, P. 2017, A\&A, 608, A72

Podolak, M., Haghighipour, N., Bodenheimer, P., Helled, R., \& Podolak, E. 2020, ApJ, 899, 45

Rosenblum, E., Garaud, P., Traxler, A., \& Stellmach, S. 2011, ApJ, 731, 66

Saumon, D., Hubbard, W. B., Chabrier, G., \& van Horn, H. M. 1992, ApJ, 391,827

Stevenson, D. J. 1977, ApJS, 35, 221

Stevenson, D. J. 1979, JPhF, 9, 791

Stevenson, D. J. 1985, Icar, 62, 4

Stevenson, D. J., \& Salpeter, E. E. 1977, ApJS, 35, 239

von Zahn, U., Hunten, D. M., \& Lehmacher, G. 1998, JGR, 103, 22815

Wahl, S. M., Hubbard, W. B., Militzer, B., et al. 2017, GeoRL, 44, 4649

Wong, M. H., Mahaffy, P. R., Atreya, S. K., Niemann, H. B., \& Owen, T. C. 2004, Icar, 171, 153 